\title{
From Thermodynamic Entropy to Knowledge Entropy
}

\author{
Constantin BRATIANU \\ Bucharest University of Economic Studies, Bucharest, Romania \\ constantin.bratianu@gmail.com
}

\begin{abstract}
The purpose of this paper is to present the evolution of the concept of entropy from engineering to knowledge management, going through information theory, linguistic entropy, and economic entropy. The concept of entropy was introduced by Rudolf Clausius in thermodynamics in 1865 as a measure of heat transfer between two solid bodies which have different temperatures. As a natural phenomenon, heat flows from the body with a higher temperature toward the body with a lower temperature. However, Rudolf Clausius defined only the change in entropy of the system and not its absolute entropy. Ludwig Boltzmann defined later the absolute entropy by studying the gas molecules behavior in a thermal field. The computational formula defined by Boltzmann relates the microstates of a thermal system with its macrostates. The more uniform the probability distribution of the microstates is the higher the entropy is. The second law of thermodynamics says that in open systems, when there is no intervention from outside, the entropy of the system increases continuously. The concept of entropy proved to be very powerful, fact for which many researchers tried to extend its semantic area and the application domain. In 1948, Claude E. Shannon introduced the concept of information entropy, having the same computational formula as that defined by Boltzmann, but with a different interpretation. This concept solved many engineering communications problems and is used extensively in information theory. Nicholas Georgescu-Roegen used the concept of entropy and the second law of thermodynamics in economics and business. Today, many researchers in economics use the concept of entropy for analyzing different phenomena. The present paper explores the possibility of using the concept of knowledge entropy in knowledge management.
\end{abstract}

Keywords: entropy, thermodynamic entropy, information entropy, knowledge entropy, order, disorder

\section{Introduction}

The industrial revolution was generated by the disruptive technologies powered by steam engines. The new technologies were based on the discovery that heat can be transformed into mechanical work by a proper design of the steam engine. Engineers made great efforts to understand the nature of heat and its transformation into mechanical work. Also, they were interested in increasing the efficiency of that transformation. Many scientists and engineers tried to understand those new phenomena and to explain them. In the beginning, heat was interpreted as a flow of a massless fluid called caloric (Handscombe\&Patterson, 2004). The difficulty of understanding the new phenomena came from the fact that scientists and engineers wanted to explain them by using the Newtonian physics, a science based on deterministic and linear thinking (Bratianu\&Vasilache, 2010), and reversible processes. Heat transfer and its transformation into mechanical work are irreversible processes and their explanation implies nonlinear and probabilistic thinking (Bratianu, 2015).

Rudolf Clausius and Lord Kelvin were among the first to understand that heat transfer from a hot body to a cold one, and heat transformation into mechanical work were complex phenomena that could not be explained in terms of the Newtonian physics anymore. They need a new perspective and new parameters to describe them. Thus, thermodynamics came as a new science focusing on thermal processes which are irreversible (Atkins, 2010). Rudolf Clausius introduced in 1865 the new concept of entropy as a state function for a thermal system undergoing a change. Then, he used the concept of entropy to formulate the second law of thermodynamics. Ludwig Boltzmann starting from a totally different perspective, that of a gas 
molecules distribution, defined the entropy as the logarithm of the total number of microstates necessary to determine a certain macrostate for the gas confined within a given space. His statistical approach was criticized in the beginning, but after some time became so famous that many scientists tried to transferred it to some other research domains. Thus, entropy was introduced by Claude Shannon (1948) in the information science by using Boltzmann formulation. More recently, Nicholas Georgescu-Roegen (1999) introduced the concept of entropy in economics. Today, the concept of entropy is used in many research domains for evaluating the probability distributions of different variables in complex systems undergoing change (Ben-Naim, 2012; Caraiani, 2018; Chalidze, 2000; Handscombe\& Patterson, 2004; Zhou et al., 2013). In the present paper, we introduce the concept of entropy in the domain of knowledge management showing its usefulness in describing the knowledge distribution within an organization. Also, knowledge entropy can be used in evaluating the process of knowledge sharing or of a knowledge transfer happening during a training program (Bratianu, 2019).

\section{Literature review}

\section{Entropy in engineering thermodynamics}

The explosive development of heat engines in the $19^{\text {th }}$ century requested a new theoretical foundation to help engineers in designing powerful and efficient engines. However, heat proved to be a new phenomenon that could not be explained by using the classical Newtonian physics. It was necessary to develop a new research domain called thermodynamics focusing on thermal processes and the transformation of thermal energy into mechanical work (Atkins, 2010). In the beginning, by using metaphorical thinking, scientists explained thermal phenomena considering the existence of a weightless and invisible fluid called caloric. After years of research and new discoveries, scientists understood that thermal phenomena are produced by thermal energy, a new form of energy that is different than mechanical energy. Also, heat represents a transfer process from a body with a higher temperature level toward a body with a lower temperature level. As Atkins (2010, p. 22) remarks, "In thermodynamics, heat is not an entity or even a form of energy: heat is a mode of transfer of energy". This process can be used metaphorically to understand now the knowledge transfer and in particular, knowledge sharing. Knowledge can be transferred only from a person with a higher level of knowing to a person with a lower level of knowing. Knowledge transfer is an irreversible process, as heat transfer is.

The transformation of thermal energy into mechanical energy, respectively the transformation of heat into mechanical work to power engines is supported by the second law of thermodynamics, a law that came to a final understanding as a result of many contributions from scientists and engineers. Lord Kelvin, formulated this law having in mind the need for two heat sources in order to produce mechanical work: "No cyclic process is possible in which heat is taken from a hot source and converted completely into work" (Atkins, 2010, p. 41). In other words, heat can generate mechanical work if the energy is transferred from a heat source with a high temperature to a heat sink with a lower temperature. Rudolf Clausius formulated the second law having in mind the heat transfer between two bodies at different temperatures: "Heat does not pass from a body at low temperature to one at high temperature without an accompanying change elsewhere" (Atkins, 2010, p. 42). Heat transfer is an irreversible process, and naturally its direction is from the body with higher temperature toward the body with lower temperature. To describe more accurately that process, Clausius introduced in 1865 the new concept of entropy 
(Atkins, 2010; Ben-Naim, 2012). The name entropy comes from Greek, where it means transformation content. Mathematically, Clausius defined the variation of entropy $(\mathrm{dS})$ of a thermal system with a variation of heat $(\mathrm{dQ})$ at a given temperature $(\mathrm{T})$ :

$\mathrm{dS}=\mathrm{dQ} / \mathrm{T}$

PICBE $\mid 591$

Here, the symbol "d" stands for a very small variation in heat and entropy, at the absolute temperature T. With the new concept of entropy, Clausius could give a unifying formulation for the second law of thermodynamics, as follows: "The entropy of the universe increases in the course of any spontaneous change" (Atkins, 2010, 49). The meaning of the "universe" in this formulation is the thermal system and its surroundings. It is important to understand the meaning of the second law of thermodynamics and that of the concept of entropy in order to understand how we can make use of the entropy in studying the processes of knowledge transfer and knowledge sharing (Cyr \&Choo, 2010; Davenport \&Prusak, 2000; Nonaka\& Takeuchi, 1995).

\section{Entropy in statistical mechanics}

While Rudolf Clausius was looking at the macroscopic world, Ludwig Boltzmann was looking at the microscopic world, and tried to find a link between the two worlds. He was interested in describing the behavior of gas molecules within a given space of confinement. He observed that each thermodynamic macrostate of a gas can be defined in terms of the probability distribution of the microstates generated by the motion of the gas molecules. Let us consider a certain volume $\mathrm{V}$ of gas, containing $\mathrm{N}$ molecules, contained in a vessel at temperature $\mathrm{T}$ and pressure $\mathrm{P}$. Let us assume that $\mathrm{W}$ "is a measure of the number of ways that the molecules of a system can be arranged to achieve the same total energy (the 'weight' of an arrangement)" (Atkins, 2010, p. 54). Thus, $\mathrm{W}$ depends on the probability distribution of the molecules at a certain moment of time within the volume $\mathrm{V}$. The correlation between the probability distribution of the $\mathrm{N}$ microstates given by $\mathrm{W}$ and the gas macrostate is represented by the entropy $\mathrm{S}$ :

$\mathrm{S}=\mathrm{k} \log \mathrm{W}$

Mathematically, it can be demonstrated that both formulations for entropy, given by Clausius and Boltzmann are equivalent. Clausius' entropy is focusing on the thermal energy transfer, while the Boltzmann's entropy is focusing on the probability distribution of the microstates for a certain macrostate of a thermal system undergoing a change.

In a solid body the degree of motion of molecules is very low, which indicates a low entropy. In a fluid, the motion of molecules is much higher and thus the fluid can flow withing a given geometry; its entropy is higher than that of a solid. In a gas, molecules have a very high degree of motion, leading to a high level of entropy. This situation made many scientists to make connections between order and disorder through entropy. Whenever the disorder of a system is increasing as a result of a change, its entropy is increasing. This idea was used by some researchers to study the entropy of different structures, including organizational arrangements.

\section{Entropy in information systems}

Claude E. Shannon studied the process of communication in technological systems and came out with the theory of information entropy (Shannon, 1948), which immediately has been adapted to 
many other research domains (Chalidze, 2000). In the beginning of the $20^{\text {th }}$ century wire communications for long distances had problems due to external perturbations, which lead to many messages distortion. Thus, the engineering problem was to find ways of reducing the effects of the external perturbations on the electrical signals used to carry on the semantic information by a coding system. R.V.L. Hartley (1928) had the idea of decoupling the meaning from the electrical signals and to measure somehow the transfer of those signals between the sender and the receiver. He suggested to use a logarithmic function for that measure, but he could not end up with a clear solution. Claude E. Shannon starting to study that problem for a simple communication system composed of a sender, a coding system, the communicant channel, $\mathrm{s}$ decoding system and the receiver. External perturbations act along the communication channel. Shannon decoupled the meaning of words from the electrical signals and focused his research only on the electrical signals which formed the messages send from the sender to receiver. In his famous papers and then the book written with Weaver, Shannon stated that: "Frequently the messages have meaning; that is, they refer to or are correlated according to some system with certain physical or conceptual entities. These semantic aspects of communication are irrelevant to the engineering problem" (Shannon, 1948, p. 379). For Shannon, the relevant aspects were related to the probability distribution of the electrical signals which were selected to form different messages. After a mathematical analysis of the engineering communication problem Shannon came out with a formula that was similar to the Boltzmann formula of entropy:

$\mathrm{H}=-\mathrm{k} \Sigma \mathrm{p}_{\mathrm{i}} \log \mathrm{p}_{\mathrm{i}}$

Where, $\mathrm{H}$ is the information entropy, $\mathrm{k}$ is a constant, and $\mathrm{p}_{\mathrm{i}}$ represents the probability of the event $\mathrm{i}$ to be produced. The logarithmic function is considered in base 2 . Thus, when the system contains only two events, and their probability appearance is equal, then $\mathrm{H}=1$ bit (bit comes from binary digits, i.e., 0 and 1 used in the Boolean logic). In this formula, events are considered the electrical signals used for sending messages across the communication channel. Shannon emphasizes that "The form of $\mathrm{H}$ will be recognized as that of entropy as defined in certain formulations of statistical mechanics where $p_{i}$ is the probability of a system being in cell $i$ of its phase space. $\mathrm{H}$ is then, for example, the $\mathrm{H}$ in Boltzmann's famous $\mathrm{H}$ theorem. We shall call $H=-k \Sigma p_{i} \log p_{i}$ the entropy of the set of probabilities $\mathrm{p}_{1}, \ldots, \mathrm{p}_{\mathrm{n}}$ " (Shannon, 1948, p. 398). Then he remarks that $\mathrm{H}$ "will play a central role in information theory as measures of information, choice, and uncertainty" (Shannon, 1948, p. 398). In conclusion, the information entropy (H) Defined by Shannon (1948) expresses a measure of a probability distribution within a finite set of events $\mathrm{N}$, and it is totally decoupled from the message meaning. This conclusion is formulated also by Bar-Hillel and Carnap (1953, p. 147): "The measures, as defined, for instance, by Shannon, have nothing to do with what these symbols symbolize, but only with the frequency of their occurrence".

Bar-Hillel and Carnap (1953) developed a theory of semantic information starting with the Shannon's formula and replacing the communication channel with a language system. They considered some language systems which "contain a finite number of individual constants which stand for individuals (things, events, or positions) and a finite number of primitive one-place predicates which designate primitive properties of the individuals" (Bar-Hillel \&Carnap, 1953, p. 156). They demonstrated that the information entropy defined by Shannon for electrical signals can be extended to a semantic system like a language, but only for systems containing a finite set 
of semantic elements, which appear in messages with some frequencies. The semantic information can be used in automation and robotics, where the language systems are finite and designed to generate a finite number of semantic combinations.

Recently, Floridi $(2005,2012,2013)$ focused on the concepts of information and information entropy and showed that researchers should be aware of the limitations introduced by the Bar-Hillel and Carnap assumptions, and the need to understand very well the context of any application: "Polysemantic concepts such as information can be fruitfully analyzed only in relation to well-specified contexts of application" (Floridi, 2005, p. 352).

\section{Entropy in organizational structures and hierarchies}

The research performed by Boltzmann on the probability distribution of gas molecules can be interpreted very well from the perspective of order and disorder within a system. Already we mentioned the fact that in solids the crystalline structure imposes a rigid arrangement of molecules such that the system entropy is very low; in liquids, the motion of molecules is allowed along the flow and the entropy is higher; in gases, molecules have a chaotic motion and the entropy of the system is very high. It increases with the gas temperature. Thus, entropy can be related to the order or disorder of the elements contained in a given structure. When the structure is changing from order to disorder, its entropy is increasing (Ben-Naim, 2012; Chalidze, 2000). Boltzmann explained the tendency of any structure toward a larger disorder, respectively, toward higher entropy, as a necessity of increasing its stability. The entropy of a system with a given structure attains a maximum value when all its microstates have the same probability distribution. "He showed that entropy is a measure of disorder in the system, that a multi-particle system tends to develop to a more probable state, and such a more probable state is a state of higher disorder. This development (toward disorder) continues until a system reaches thermodynamic equilibrium, which is the highest state of disorder for any given system" (Chalidze, 2000, p. 11). However, we must admit that order and disorderare relative concepts and sometimes very fuzzy since it is almost impossible to define precisely a state of order or disorder in any given system. "Disorder is a highly relative, if not wholly improper concept: something is in disorder only with respect to some objective, nay, purpose" (Georgescu-Roegen, 1999, p. 142).

When Taylor (1998) started to transfer rules and methods from engineering to management, he wanted actually to increase the order in organization for the benefit of control and increased efficiency. During industrial era, organizations were conceives as machines: "We talk about organizations as if they were machines, and as a consequence, we tend to expect them to operate as machines: in a routinized, efficient, reliable, and predictable way" (Morgan, 1997, p. 13). This organization model is based on linear thinking (Bratianu\&Vasilache, 2010). The low entropy of such an organizational structure has a negative influence on innovation and agility of the company. In the context of a turbulent business environment it is necessary to increase the flexibility of the organizational structure by flattening the hierarchy and reducing the centralized control (Bratianu, Vasilache\&Jianu, 2006; Hatch, 997). We may say that management has been created in order to control the organizational entropy. The lower the organizational entropy is, the better and easier control, but low stimulation for innovation. The higher the organizational entropy, the more flexibility and agility, and a better climate for stimulating knowledge sharing and innovation (Nonaka, Toyama\& Hirata, 2008; Yonghi et al., 2013). The entropy of any organization increases during changes, like mergers and acquisitions (Bratianu\&Anagnoste, 2011).

DOI: 10.2478/picbe-2020-0055, pp. 589-596, ISSN 2558-9652| Proceedings of the $14^{\text {th }}$ International Conference on Business Excellence 2020 


\section{Knowledge entropy}

Knowledge transfer, in all its forms and manifestations, is a key process in knowledge management. Among the most important forms of knowledge transfer we mention knowledge sharing (Cyr \&Choo, 2010; Leistner, 2010; Nonaka\& Takeuchi, 1995), and intergenerational knowledge transfer (Bratianu et al., 2011; Lefter et al., 2011).Although most of the researchers refer only to the explicit knowledge that is rational, the process of knowledge transfer should be considered in its complexity of interaction of all three fundamental fields of knowledge, i.e., rational, emotional and spiritual knowledge (Bratianu, 2018a; Bratianu\&Bejinaru, 2019, 2020).

We introduce the concept of knowledge entropy based on the analogy with the thermodynamics phenomena presented above and its capacity to explain knowledge transfer and to measure the complexity of knowledge management through the probability of knowledge distribution. Based on the information entropy formula developed by Shannon (1948), we define knowledge entropy $(K E)$ as follows:

$\mathrm{KE}=-\mathrm{C} \Sigma \mathrm{p}_{\mathrm{i}} \log \mathrm{p}_{\mathrm{i}}$

Here, $\mathrm{C}$ is a constant that is an arbitrary positive number chosen to adjust the measurement to a certain scale, and a given context. Considering all employees as knowledge owners or knowledge sources, we may assume that $\mathrm{p}_{1}, \ldots, \mathrm{p}_{\mathrm{n}}$ represents the knowledge distribution within the company. That distribution can be considered in space, or in time. Knowledge entropy attains its maximum value when all these probabilities are equal, which will give a knowledge dynamics equilibrium. However, such a situation cannot be found in reality since individuals have different experiences and different emotional and spiritual states. Because in knowledge management there is no metric to measure absolute knowledge for each individual, we shall use relative values for knowledge, defined with respect to a certain level of knowing.

The concept of knowledge entropy can be very useful in measuring the efficiency of knowledge transfer during training programs, or for knowledge sharing processes within companies or communities of practice (Wenger, 1998). Also, by measuring the knowledge distribution within a given department or company, managers can make decisions concerning their strategies for increasing knowledge entropy, which leads to enhancing innovation. Also, by measuring knowledge entropy researchers can show the ways for developing intellectual capital and creating intelligent organizations (Bratianu, 2018b; Bratianu, 2013).

\section{Conclusions}

Entropy is a very powerful concept that can be used in many research domains for measuring different probability distributions. Introduced by Clausius in thermodynamics, and then developed by Boltzmann for statistical mechanics, entropy became a well-known concept especially after the mathematical theory of communication developed by Shannon. Thus, information entropy stimulated researchers from many fields of science and engineering to find new applications for it and for evaluating change processes based on the second law of thermodynamics. 
The present paper focuses on the different methods of using the concept of entropy, and the practical ways of measuring it. We introduce for the first time the concept of knowledge entropy to be used in knowledge management, especially for understanding the processes of knowledge transfer and knowledge dynamics within a given organization. The concept is based on relative measures of knowledge and the knowledge distribution within a given space or time in a well-defined context.

PICBE | 595

\section{References}

Atkins, P. (2010). The laws of thermodynamics: a very short introduction. Oxford, UK: Oxford University Press.

Bar-Hillel, Y. \&Carnap, R. (1953).Semantic information.The British Journal for the Philosophy of Science, 4(14), 147-157.

Ben-Naim, A. (2012). Entropy and the second law: interpretations and miss-interpretations. London, UK: World Scientific.

Bratianu, C. (2013). Nonlinear integrators of the organizational intellectual capital.In Fathi, M. (Ed.).Integration of practice-oriented knowledge technology: trends and perspectives, pp. 3-17. Heidelberg: Springer.

Bratianu, C. (2015). Developing strategic thinking in business education.Management Dynamics in the Knowledge Economy, 3(3), 409-429.

Bratianu, C. (2018a). A holistic view of the organizational knowledge dynamics.HOLISTICA Journal of Business and Public Administration, 9(2), 7-22.

Bratianu, C. (2018b). Intellectual capital research and practice: 7 myths and one golden rule. Management \& Marketing. Challenges for the Knowledge Society, 13(2), 859-879.

Bratianu, C. (2019). Exploring knowledge entropy in organizations.Management Dynamics in the Knowledge Economy, 7(3), 353-366.

Bratianu, C. \&Anagnoste, S. (2011). The role of transformational leadership in mergers and acquisitions in emergent economies.Management \& Marketing, 6(2), 319-326.

Bratianu, C. \&Bejinaru, R. (2019). The theory of knowledge fields: a thermodynamic approach. Systems, 7(2), 20, 1-12.

Bratianu, C. \&Bejinaru, R. (2020). Knowledge dynamics: a thermodynamic approach. Kybernetes, 49(1), 6-21.

Bratianu, C. \&Vasilache, S. (2010). A factorial analysis of the managerial linear thinking model.International Journal of Innovation and Learning, 8(4), 393-407.

Bratianu, C., Agapie, A., Orzea, I. \&Agoston, S. (2011). Intergenerational learning dynamics in universities.Electronic Journal of Knowledge Management, 9(1), 10-18.

Bratianu, C., Vasilache, S. \&Jianu, I. (2006).Business management. Bucharest: Editura ASE.

Caraiani, P. (2018). Modeling the comovement of entropy between financial markets.Entropy, 20(6), 417.

Chalidze, V. (2000).Entropy demystified: potential order, life and money. Portland, OR: Universal Publishing. 
Cyr, S. \&Choo, C.W. (2010). The individual and social dynamics of knowledge sharing: an exploratory study. The Journal of Documentation, 66(6), 824-846.

Davenport, T.H. \&Prusak, L. (2000).Working knowledge: how organizations manage what they know. Boston, MA: Harvard Business School Press.

Floridi, L. (2005). Is semantic information meaningful data? Philosophy and Phenomenological Research, 70(2), 351-370.

PICBE | 596

Floridi, L. (2012). Semantic information and the network theory of account.Synthese, 184(3), 431-454.

Floridi, L. (2013). The philosophy of information. Oxford, UK: Oxford University Press.

Georgescu-Roegen, N. (1999). The entropy law and the economic process. Cambridge, MA: Harvard University Press.

Handscombe, R.D. \& Patterson, E.A. (2004).The entropy vector: connecting science and business. London, UK: World Scientific.

Hartley, R.V.L. (1928). Transmission of information.Bell System Technical Journal, 7, 535-563.

Hatch, M.J. (1997).Organization theory: modern, symbolic, and postmodern perspectives. Oxford, UK: Oxford University Press.

Lefter, V., Bratianu, C., Agapie, A., Agoston, S. \&Orzea, I. (2011).Intergenerational knowledge transfer in the academic environment of the knowledge-based economy.Amfiteatru Economic Journal, 13(30), 392-403.

Leistner, F. (2010).Mastering organizational flow: how to make knowledge sharing work. New York, NY: John Wiley \& Sons.

Morgan, G. (1997). Images of organizations. ${ }^{\text {nd }}$ Edition. London, UK: SAGE Publications.

Nonaka, I. \& Takeuchi, H. (1995).The knowledge-creating company. How Japanese companies create the dynamics of innovation. Oxford, UK: Oxford University Press.

Nonaka, I., Toyama, R. \& Hirata, T. (2008).Managing flow: a process theory of the knowledgebased firm. Houndmills, UK: Palgrave Macmillan.

Shannon, C.E. (1948). A mathematical theory of communication.Bell System Technical Journal, 27(3), 379-423.

Shannon, C.E. \& Weaver, W. (1949).The mathematical theory of communication. Champaign, IL: University of Illinois Press.

Taylor, F.W. (1998). The principles of scientific management. Mineola, NY: Dover Publications.

Yonghi, L., Wu, C., Luo, P. \& Zhang, W. (2013). Exploring the characteristics of innovation adoption in social networks: structure, homophily, and strategy. Entropy, 15(7), 26622678.

Wenger, E. (1998). Communities of practice: learning, meaning, and identity. Cambridge, UK: Cambridge University Press.

Zhou, R., Cai, R. \& Tong, G. (2013). Applications of entropy in finance: a review. Entropy, 15(11), 4909-4931. 